\title{
Nitric Oxide as a Mediator for Defense Responses
}

\author{
Diana Bellin, ${ }^{1}$ Shuta Asai, ${ }^{2}$ Massimo Delledonne, ${ }^{1}$ and Hirofumi Yoshioka ${ }^{2}$ \\ ${ }^{1}$ Biotechnology Department, University of Verona, 37134 Verona, Italy; ${ }^{2}$ Laboratory of Defense in Plant-Pathogen Interactions, \\ Graduate School of Bioagricultural Sciences, Nagoya University, Chikusa, Nagoya 464-8601, Japan
}

Submitted 8 September 2012. Accepted 2 November 2012.

Sequential recognition of invading microbes and rapid induction of plant immune responses comprise at least two recognition systems. Early basal defenses are initiated by pathogen-associated molecular patterns and pattern recognition receptors (PRR) in the plasma membrane. Pathogens produce effectors to suppress defense but plants, in turn, can sense such effectors by dominant plant resistance $(R)$ gene products. Plant PRR and R proteins modulate signaling networks for defense responses that rely on rapid production of reactive nitrogen species (RNS) and reactive oxygen species (ROS). Recent research has shown that nitric oxide (NO) mainly mediates biological function through chemical reactions between locally controlled accumulation of RNS and proteins leading to potential alteration of protein function. Many proteins specifically regulated by NO and participating in signaling during plant defense response have been identified, highlighting the physiological relevance of these modifications in plant immunity. ROS function independently or in cooperation with NO during defense, modulating the RNS signaling functions through the entire process. This review provides an overview of current knowledge about regulatory mechanisms for NO burst and signaling, and crosstalk with ROS in response to pathogen attack.

Nitric oxide (NO) mediates signaling during several physiological processes and stress responses in cells (Besson-Bard et al. 2008). Among them, NO has been shown to behave as a crucial component of plant immune response, and it participates, in cooperation with $\mathrm{H}_{2} \mathrm{O}_{2}$, in activating hypersensitive response (HR) cell death during incompatible plant-pathogen interactions (Delledonne et al. 1998; Durner et al. 1998; Yoshioka et al. 2011). NO is a highly diffusible and reactive gaseous radical endogenously produced by plants. Importantly for its signaling function, its lipophilic properties allow NO to cross plant membranes easily, but how far it can really diffuse within and between cells is still unknown. Its reactivity is also of crucial importance for its signaling functions. NO derivatives are collectively called reactive nitrogen species (RNS)

Current address for S. Asai: Sainsbury Laboratory, John Innes Centre, Norwich NR4 7UH, U.K.

Corresponding author: H. Yoshioka; Telephone and Fax: +81-52-7894283; E-mail: hyoshiok@agr.nagoya-u.ac.jp

* The $\boldsymbol{e}$-Xtra logo stands for "electronic extra" and indicates Figures 1 and 2 appear in color online.

C 2013 The American Phytopathological Society that comprise the radical $\mathrm{NO}^{\circ}$, its nitrosonium $\left(\mathrm{NO}^{+}\right)$and nitroxyl ions $\left(\mathrm{NO}^{-}\right)$, and the products of reaction between the NO radical and reactive oxygen species (ROS). When both ROS and NO accumulate, such as during recognition of plant pathogens, diffusion-limited reaction of $\mathrm{NO}$ with superoxide $\left(\mathrm{O}_{2}^{-}\right)$results in generation of peroxynitrite $\left(\mathrm{ONOO}^{-}\right)$. $\mathrm{NO}$ can also react with $\mathrm{O}_{2}^{-}$-generating $\mathrm{NO}_{\mathrm{x}}$ compounds, such as $\mathrm{NO}_{2}$, $\mathrm{N}_{2} \mathrm{O}_{3}$, and $\mathrm{N}_{2} \mathrm{O}_{4}$. All these compounds in biological systems can react with thiols or are further modified to finally produce $\mathrm{NO}_{2}^{-}$or $\mathrm{NO}_{3}^{-}$(Wendehenne et al. 2001). Here, we provide an overview of current knowledge about NO and the cross-reacting ROS function during defense responses in plants.

\section{NO-generating systems.}

In animals, NO is generated mainly by nitric oxide synthase (NOS). In plants, no direct homolog of any animal NOS gene has been found in any fully sequenced higher plant genome, even though a close homolog of animal NOS has been found in the unicellular green alga Ostreococcus tauri (Foresi et al. 2010). Nevertheless, NO can be produced by plant extracts incubated with NOS substrate arginine. Therefore, an NOS-like activity exists in plants (Asai and Yoshioka 2009; Chaki et al. 2009a; Corpas et al. 2006). This activity requires the same cofactors (NADPH, calcium, calmodulin, flavin adenine dinucleotide, flavin mononucleotide, and tetrahydrobiopterin $\mathrm{BH}_{4}$ ) as animal NOS (Asai et al. 2010a; Corpas et al. 2009) and is often referred to as the major source of $\mathrm{NO}$ in plants during plant-pathogen interactions; however, lack of identification of its enzymatic source and of proper genetic material has not allowed a final unequivocal clarification of its participation in plant defense (Gaupels et al. 2011a). In addition to this NOSlike activity, other oxidative enzymatic routes for NO production have been postulated, including oxidative reactions of polyamines (Tun et al. 2006) or hydroxylamines (Rumer et al. 2009), the natural occurrence of which has not been definitely proven in plants. Recently, a copper amine oxidase of Arabidopsis thaliana was also identified as a candidate for $\mathrm{NO}$ production from polyamines induced by abscissic acid (Wimalasekera et al. 2011). Nevertheless, the biological relevance of these findings in the context of plant defense has not been investigated.

As an alternative to oxidative routes, several reductive enzymatic systems using nitrite as a substrate for NO production were also reported for plants; however, although the described oxidative pathways are normally active under normoxia, reductive pathways usually become major NO-generating mechanisms when oxygen decreases (Gupta et al. 2011). For instance, nitrite reduction is catalyzed by a mitochondrial electron transport chain in the mitochondrial inner membrane under the condition of oxygen deprivation, probably via cytochrome c oxi- 
dase or reductase (Gupta and Igamberdiev 2011; Planchet et al. 2005). Another enzymatic activity using nitrite as a substrate to generate NO has been reported for tobacco roots in which the activity is probably derived from a plasma membrane-bound nitrite:NO-reductase (Ni-NOR) (Stohr et al. 2001). This enzyme, which is root specific, seems to mainly participate in sensing nitrate availability in soil and in regulating root infection by mycorrhizal fungi and, therefore, seems to not directly participate in NO production during pathogen infection (Moche et al. 2010). Another alternative candidate enzyme for NO production from nitrite is the peroxisomal xanthine oxidoreductase (XOR) that catalyzes NO production only under strict anaerobic conditions, thus strongly limiting its possible relevance for NO signaling in defense (Godber et al. 2000). By contrast, NO can clearly be produced, even in normoxic conditions, by the enzyme nitrate reductase (NR) (Yamasaki et al. 1999). NR catalyzes the reduction of nitrate to nitrite in an $\mathrm{NAD}(\mathrm{P}) \mathrm{H}$-dependent manner. In addition, NR is capable of reducing nitrite to NO (Bright et al. 2006; Rockel et al. 2002). According to its enzymatic properties, the efficiency of this reaction in vivo could be limited by higher concentration of nitrate compared with nitrite, although the reaction efficiency could increase with an increase in nitrite concentration (Rockel et al. 2002).

In Arabidopsis, NR is encoded by genes NIA1 and NIA2, and the activity of NR proteins is tightly regulated in response to several environmental factors. Regulation occurs at the expression level and is mediated by substrate availability by binding 14-3-3 proteins and by post-translational modifications, such as phosphorylation and redox regulation (Hardin et al. 2009; Lillo et al. 2004; Wang et al. 2010). NR also undergoes sumoylation by AtSIZ1, an E3 SUMO ligase, and this modification dramatically increases its enzymatic activity, controlling nitrogen assimilation as well as NO content (Park et al. 2011). By genetic approaches using a nialnia2 double mutant line (Wilkinson and Crawford 1993), NR has been shown to participate in NO production in several physiological contexts, such as abscissic acid-induced stomatal closure, hypoxia, and cold stress, or in nitrogen-fixing nodules (Cantrel et al. 2011; Desikan et al. 2002; Dordas et al. 2004; Horchani et al. 2011; Zhao et al. 2009). Importantly, NR participates in NO accumulation during defense responses to necrotrophic fungal pathogens (Asai et al. 2010b; Perchepied et al. 2010) and bacteria

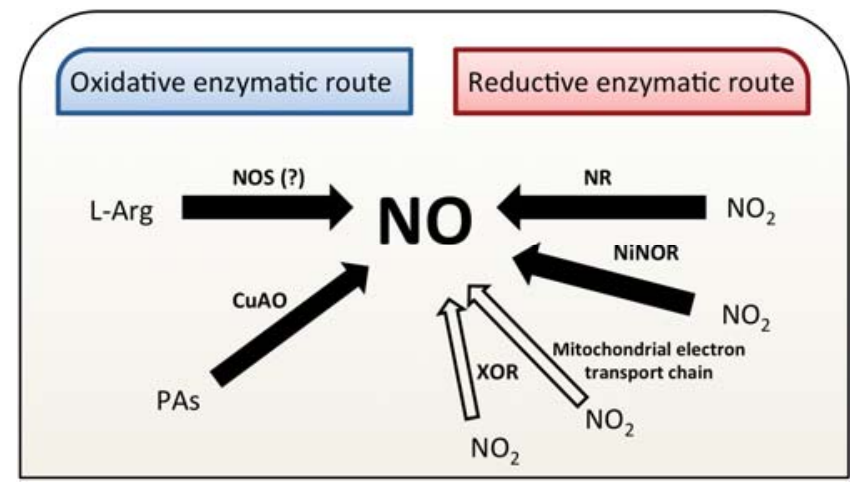

Fig. 1. Oxidative and reductive routes for nitric oxide (NO) synthesis in plants. NO can be produced from nitrite via reductive enzymatic routes, catalyzed by nitrate reductase (NR) or nitrite-NO reductase (Ni-NOR) in roots. Additional reductive enzymatic routes for $\mathrm{NO}$ synthesis from $\mathrm{NO}_{2}$ mediated by the mitochondrial electron transport chain or peroxysomial xanthine oxidoreductase (XOR) exist but typically occur under anoxic conditions (white arrows). Oxidative NO biosynthetic pathways include the nonidentified nitric oxide synthase (NOS)-like enzyme, and NO production from polyamines (PAs) mediated by enzymes, such as copper amine oxidase $(\mathrm{Cu} \mathrm{AO})$.
(Modolo et al. 2006; Oliveira et al. 2009), or upon perception of chemical elicitors from fungal plant pathogens or other pathogen-associated molecular patterns (Rasul et al. 2012; Shi and Li 2008; Yamamoto-Katou et al. 2006). However, by using electron paramagnetic resonance to detect NO production, Modolo and associates (2005) showed that foliar extracts of the mutant nialnia2 still have the capacity to produce NO, like wild-type plants, upon pathogen infection when nitrite is exogenously supplied. This result suggests that additional mechanisms to reduce nitrite into NO exist, and that the reported decreased capability of NO synthesis of nialnia2 mutant plants with defective NR activity upon pathogen infection is likely an indirect effect due to the reduced nitrite content of this line. A further argument in favor of additional, still undiscovered, NO production systems in defense was from the study of Rasul and associates (2012), in which oligogalacturonides induced NO production in Arabidopsis. This NO production was not completely suppressed using either an L-arginine-dependent NO synthesis inhibitor or genetic or pharmacological approaches to impair NR expression and activity and, therefore, further supports the possible existence of an alternative route to the NR- and arginine-dependent pathways for NO production during defense. In conclusion, differently from animals, a plethora of mechanisms have been reported for NO production in plants in addition to NOS activity (Fig. 1), although their relative participation and importance for defense against pathogens has not yet been fully clarified.

However, recent findings in plant NO research field provide some important information about upstream signaling cascades from pathogen perception to induction of NO production, as well as of production of ROS, acting together and modulating NO activity. In Nicotiana benthamiana, gain-of-function and loss-of-function analyses showed that mitogen-activated protein kinase (MAPK) cascade MEK2-SIPK/NTF4 controls NO and ROS production induced by elicitor INF1, with the latter modulated also by MEK1-NTF6 cascade. Similarly, NO accumulation induced by fungal elicitor $\mathrm{Nep} 1_{\mathrm{Mo}}$ was mediated by the same MAPK-signaling cascade. By using NOS or NR inhibitors, NOS-like activity and NR activity participated in NO burst induced by elicitor INF1 or by constitutively active MEK2. By silencing $N b R B O H B$, ROS production induced by INF1 depended on plasma membrane NADPH oxidase (Asai and Yoshioka 2009; Asai et al. 2008; Yoshioka et al. 2011, Zhang et al. 2012). Finally, by using virus-induced gene silencing (VIGS), flavin biosynthesis enzyme RibA participated in regulating NO and ROS production induced by constitutively active MEK2, probably because of its participation in biosynthesis of prostetic groups important for NOS, NR, and RBOH activities (Asai et al. 2010a). Interestingly, MPK6, the Arabidopsis ortholog of SIPK, interacts in vitro and in vivo with NIA2 and phosphorylates NIA2 at a specific serine residue, leading to an increase in NR activity and NO biosynthesis in response to $\mathrm{H}_{2} \mathrm{O}_{2}$ during Arabidopsis root development (Wang et al. 2010). According to these results, and given the high conservation of docking sequence and phosphorylation sites in NR proteins, NR may share with mammalian NOS a regulatory mechanism based on phosphorylation at a specific serine residue (Wang et al. 2011). Nevertheless, the possible relevance of this specific regulation for NO production activity in the context of plant defense has not been investigated.

In addition to MAPK, other signaling molecules participating in the signaling pathway leading to NO production during defense are currently emerging. For example, an exogenous treatment with extracellular adenosine triphosphate (eATP), a regulator of pathogen defense in plants, induces phosphatidic acid accumulation and NO synthesis in tomato cultured cells, and this NO synthesis is dependent on phosphatidic acid and $\mathrm{Ca}^{2+}$ 
influx (Sueldo et al. 2010). Nevertheless, more recent data question the participation of NO, at least in the eATP-induced cell death process (Sun et al. 2012). Cytosolic calcium increase is well known as an essential early event in pathogen-induced immune signaling acting upstream of NO but the molecular events mediating it and linking it to NO increase are not fully understood. Recent data indicate that the cyclic nucleotide gated channel 2 (CNGC2), a nonselective cation-conducting channel activated by cyclic nucleotides, is a possible candidate mediating this event (Ali et al. 2007). By using inhibitors of adenylyl cyclase or phosphodiesterase, the enzymes responsible for cAMP synthesis and degradation, Ma and associates (2009) showed participation of this cyclic nucleotide in NO generation through $\mathrm{CNGC} 2$ and $\mathrm{Ca}^{2+}$ influx regulation. Recently, Qi and associates (2010) identified receptor AtPepR1 participating in pathogen defense that includes a cyclase domain. They showed that the specific ligand AtPep3 induces a cytosolic calcium increase dependent on the receptor and on CNGC2. Even if the receptor cyclase activity has been shown only in vitro and is substantially lower compared with cyclase activity reported for animal cells, they speculated that the receptor could activate CNGC2 through its cyclase activity. These data seems to suggest that cyclic nucleotide signaling, which is traditionally regarded as acting downstream of $\mathrm{NO}$, could also participate in regulation of NO biosynthesis during defense.

\section{NO signaling.}

The best-characterized signaling pathway through which NO exerts its action in animals is mediated by second messenger cGMP. NO interacts with the heme ferrous iron of the enzyme soluble guanylate cyclase (sGC) to activate it and, thus, lead to cGMP production. In plants, pathogen infections and NO accumulation can also lead to cGMP production, but plant enzyme(s) responsible for the production have not been identified (Isner and Maathuis 2011; Meier et al. 2009). Only recently, by using a GC- and NO-binding domain search, an NO-sensitive GC enzyme has been identified in A. thaliana. Nevertheless, its predicted GC activity has been confirmed only in vitro, and its possible relevance for NO signaling in plants still awaits demonstration (Mulaudzi et al. 2011). NO signaling acts through MAPK. For example, different hormone signaling in different physiological contexts, as well as in cell death (Clarke et al. 2000; Pagnussat et al. 2004). NO signaling can directly regulate MAPK activity, as shown for the animal system, or act through interaction of MAPK with other proteins that are directly modified by NO (Wawer et al. 2010). Finally, reports exist providing evidence of a role for other second messengers, such as calcium and phosphatidic acid, that were also involved in NO biosynthesis in mediating NO downstream signaling in defense (Laxalt et al. 2007; Raho et al. 2011). However, the best-characterized signaling mechanism through which NO exerts its function in plants during defense is based on its chemical reaction with proteins (Leitner et al. 2009).

\section{$S$-nitrosylation of NO sensor proteins.}

$S$-nitrosylation consists of the covalent addition of an NO moiety to the sulfhydryl group of cysteine residues in target proteins, forming $S$-nitrosothiol (SNO) (Lindermayr and Durner 2009). This modification does not generically target all cysteine residues of proteins but specifically affects a subset of cysteine sensor residues that, given their molecular environment, shows higher susceptibility to oxidation. These particularly reactive cysteine residues can undergo different redox-based post-translational modifications, including $S$-nitrosylation, to sense redox changes such as those that typically occur during biotic stresses. Modification of key regulatory proteins affect their activities, localization, interaction with other proteins, and stability (Yun et al. 2012). Motif-based computational identification of cysteines that can undergo $S$-nitrosylation is not straightforward but different predictive methods have been developed that can be used with some success to identify putative targets also in plants (different computational strategies are discussed by Fares and associates [2011] and Weerapana and associates [2010]).

The level of SNO in plant cells is controlled by both NO content and turnover of $S$-nitrosoglutathione (GSNO) that performs protein trans-nitrosylation but also acts as an NO-bioreservoir (Zhang and Hogg 2004). This turnover is regulated by GSNO reductase (GSNOR), an evolutionarily conserved enzyme that controls intracellular levels of GSNO and, indirectly, $S$-nitrosylated proteins in many eukaryotes (Liu et al. 2001). In animals, in addition to GSNOR, thioredoxins or thioredoxin reductases can also directly degrade SNO to modulate the SNO content (Benhar et al. 2008), and evidence of their participation in denitrosylation reactions during defense response has emerged recently for plants as well (Spoel and Loake 2011; Tada et al. 2008). Plants with impaired GSNO metabolism are compromised in several physiological processes (Kwon et al. 2012; Lee et al. 2008), indicating that fine-tuned control of SNO homeostasis is important for growth, development, and resistance to abiotic and biotic stresses. Interestingly, some contradictory results have shown correlation between GSNO content and plant disease resistance or cell death (Chen et al. 2009; Espunya et al. 2012; Feechan et al. 2005; Holzmeister et al. 2011; Rusterucci et al. 2007; Yun et al. 2011). In any case, the levels of GSNO increase during the establishment of plant disease resistance and alterations in the GSNO content, by either genetic or pharmacological approaches, strongly affect defense responses and cell death, as well as salicylic acid (SA) signaling and cross-talk with other hormones participating in defense.

Several proteomic studies have identified numerous candidate proteins that undergo $S$-nitrosylation in plants. The physiological relevance of this modification for many of these candidates has been investigated (Astier et al. 2011, 2012; Terrile et al. 2012; van der Linde et al. 2011; Yun et al. 2011). Defense-related proteins regulated by $S$-nitrosylation are either enzymes or transcriptional activators or co-activators participating in plant immunity or HR cell death.

Among them, the nonexpressor of NPR1 (pathogenesisrelated (PR)1) has long been known to act as a master positive regulator in plant immunity, co-activating defense gene expression and key components of SA-mediated signal transduction. Recently, Wu and associates (2012) showed this protein to directly bind SA, working as an SA receptor. NPR1 undergoes redox regulation and $S$-nitrosylation that control its activity (Mou et al. 2003; Tada et al. 2008). In unchallenged cells, NPR1 oligomerization through intermolecular disulfide bridges sequesters NPR1 in the cytoplasm. As a consequence of redox changes induced by pathogen inoculation and of SA accumulation, the protein is reduced, monomerized, and translocated to the nucleus, where it upregulates a set of disease resistance genes. $S$-nitrosylation was first shown to promote disulfide bond formation and oligomerization (Tada et al. 2008). In addition, Lindermayr and colleagues (2010) showed that treatment with GSNO promotes translocation of NPR1 into the nucleus. Novel research is expected to clarify cross-talk between NO and SA signaling and these apparently contradictory data. In the nucleus, NPR1 interacts with the TGACGmotif binding factor (TGA) that binds to elements of the PR1 promoter, promoting $P R$ gene expression and defense. TGA is also redox regulated and $S$-nitrosylated. Its oxidized form carries disulfide bonds, precluding its interaction with NPR1. GSNO-mediated $S$-nitrosylation of cysteine residues partici- 
pating in disulfide bond formation protects TGA from oxidative modification, resulting in increased DNA-binding activity and stability of TGA when NPR1 is translocated into the nucleus (Lindermayr et al. 2010).

Interestingly, AtSABP3 (A. thaliana SA binding protein 3) shows SA binding properties, and carbonic anhydrase activity required to express resistance against pathogens undergoes $S$ nitrosylation during HR (Wang et al. 2009). S-nitrosylation suppresses both binding of SA and its carbonic anhydrase activity. Inhibition of AtSABP3 CA functioning by $S$-nitrosylation could contribute to a negative feedback loop that modulates plant defense response and cell death (Wang et al. 2009). In any case, this finding further reinforces the connection between NO and SA in plant defense.

Glyceraldehyde 3-phosphate dehydrogenase (GAPDH) is an important glycolytic enzyme. Like NPR1, this enzyme undergoes a complex redox regulation and its activity is inhibited by oxidation, $S$-nitrosylation, and nitration. In animals, GAPDH binds to and stabilizes Siah1 (an E3 ubiquitin ligase) upon $S$ nitrosylation, promoting its translocation to the nucleus, where it degrades nuclear proteins, finally promoting apoptosis. New studies are proposing now that GAPDH could act as a nuclear nitrosylase, propagating by trans-nitrosylation NO signaling within defined cellular pathways (Kornberg et al. 2010; Stamler and Hess 2010). Accordingly, trans-nitrosylation by GAPDH of nuclear substrates histone deacetylase 2 or class III deacetylase sirtuin-1 modulate transcription (Hess and Stamler 2012; Sen and Snyder 2011). In plants, isoforms NtGAPCa and NtGAPCb in tobacco cells constitutively interact with salt-stress-activated protein kinase NtOSAK, and $S$-nitrosylation of these GAPDH enzymes promotes translocation into the nucleus of the complex (Wawer et al., 2010). Therefore, because plant GAPDH also can be $S$-nitrosylated and can relocalize to the nucleus, an event similar to that in the animal system seems possible for plants (Holtgrefe et al. 2008; Romero-Puertas et al. 2008).
Pro-metacaspase 9 (pMC9) is a relative of animal caspases acting as programmed cell death executioners, which are $S$-nitrosylated cysteines located at the active site. These modifications keep pMC9 inactive under normal conditions, inhibiting its autoprocessing activity, similar to mammalian caspases that are also $S$-nitrosylated in inactive resting cells. In this case, differently from previously discussed $S$-nitrosylated proteins, signaling promotes denitrosylation that actively triggers cell death (Belenghi et al. 2007).

NO directly modulates its reactivity by $S$-nitrosylation of ROS producers and ROS or RNS scavengers. NADPH oxidase AtRBOHD is a major participant in ROS production induced by pathogens in Arabidopsis (Torres et al. 2005). Yun and associates (2012) showed $S$-nitrosylation of Arabidopsis AtRBOHD and the consequences for its ROS production activity; cysteine $890 S$-nitrosylation of AtRBOHD during defense decreases NADPH oxidase activity and then reduces ROS accumulation, limiting the later stages of $\mathrm{HR}$ cell death. However, peroxiredoxin II E (PrxII E) detoxifies $\mathrm{H}_{2} \mathrm{O}_{2}$ and $\mathrm{ONOO}^{-}$under normal conditions, and its activity is inhibited by $S$-nitrosylation. During $\mathrm{HR}, S$-nitrosylation of PrxII E would increase $\mathrm{ONOO}^{-}$ availability and related signaling by inhibiting the activity of its detoxifying enzyme (Romero-Puertas et al. 2007).

In conclusion, NO signaling during defense response mediated by cysteine $S$-nitrosylation includes modification of different target regulatory proteins participating in plant defense, leading to either protein relocalization or activity modulation in which all proteins finally contribute, even if in different ways, to the development and control of defense in plants (Fig. 2).

\section{Protein tyrosine nitration by $\mathrm{ONOO}^{-}$.}

$\mathrm{ONOO}^{-}$is formed in a diffusion-controlled reaction between stoichiometric amounts of NO and superoxide, both of which accumulate during HR. Accumulation of $\mathrm{ONOO}^{-}$during biotic stress has been confirmed by using specific fluorescent dye

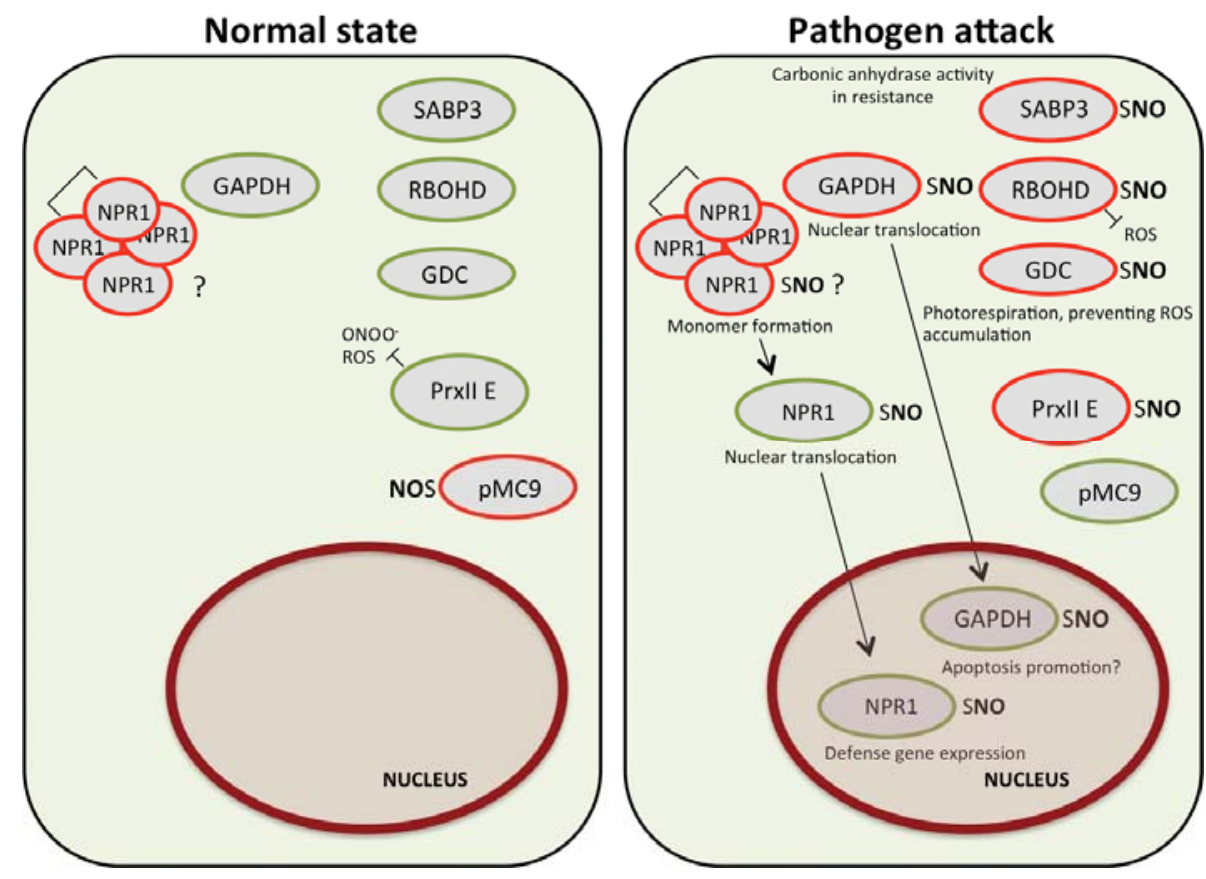

Fig. 2. Cysteine $S$-nitrosylation controls the activity of regulatory proteins participating in plant defense by either promoting (green) or inhibiting (red) their activity. Under physiological conditions, $S$-nitrosylation inhibits prometacaspase 9 (pMC9), a cysteine protease participating in hypersensitive response (HR) cell death (left panel). Induced nitric oxide (NO) production during defense (right panel) leads instead to $S$-nitrosylation of non-expressor of NPR1 monomer, glyceraldehyde 3-phosphate dehydrogenase (GAPDH), salicylic acid binding protein 3 (SABP3), peroxiredoxin II E (PrxII E), respiratory burst oxidase homolog D (RBOHD), and glycine decarboxylase (GDC). $S$-nitrosylation of the transcriptional co-activator NPR1 promotes NPR1 nuclear translocation and expression of defense genes. Similar to NPR1, GAPDH $S$-nitrosylation also promotes nuclear translocation. Normal GAPDH activity is blocked upon $S$-nitrosylation, as well as SABP3, PrxII E, RBOHD, and GDC activities. NPR1 and SABP NO sensor proteins participate more in defense establishment and immune signaling, and the other indicated sensor proteins, whose activity is regulated by NO, directly modulate HR cell death. 
HKGreen-2 in Arabidopsis leaf discs challenged with the avirulent pathogen Pseudomonas syringae pv. tomato carrying the $A v r B$ gene (Gaupels et al. 2011b), which agreed with previous results obtained with a less specific APF dye (Saito et al. 2006). $\mathrm{ONOO}^{-}$is a strong oxidant agent that leads to cell death in mammals but not in plants (Delledonne et al. 2001), and it is now widely recognized to exert important signaling functions by nitrating proteins (Arasimowicz-Jelonek and FloryszakWieczorek 2011; Vandelle and Delledonne 2011).

Tyrosine nitration consists of adding a nitro group to the ortho-position of the aromatic ring of tyrosine residues, forming 3-nitrotyrosine. This kind of protein modification appears to occur selectively, because only a limited number of proteins and tyrosines are targeted by nitration. Despite this selectivity, a consensus primary sequence for tyrosine nitration has not been defined. Thus, nitration may depend on a secondary or higher-order structure and is promoted by exposure of the aromatic ring on the surface of the protein, proximity to negatively charged residues, and the absence of nearby cysteine residues, although this last criterion is still debatable (Bayden et al. 2011). Nitration can alter protein conformation and impose steric restriction, thus influencing protein structure and activity. In animals, depending on the protein, tyrosine nitration has either a stimulatory or an inhibitory effect (Radi 2004) on denitrase activity, which would account for reversibility of this modification; however, the corresponding enzyme has not been isolated (Gorg et al. 2007).

In plants, investigation of the functional impact of tyrosine nitration is just at the beginning. For a long time, protein tyrosine nitration has been considered to be a marker for nitrosative stress. However, the observed correlation between $\mathrm{ONOO}^{-}$ accumulation and the transient increase in tyrosine-nitrated proteins during HR (Romero-Puertas et al. 2007), the selectivity and putative reversibility of this post-translational modification (Alvarez et al. 2011; Melo et al. 2011), and the identification of several putative target proteins undergoing tyrosine nitration under physiological conditions (Chaki et al. 2009b; Lozano-Juste et al. 2011) suggest a signaling role.

By using a proteomic approach, eight target proteins participating in photosynthesis, ATP synthesis, the Calvin cycle, glycolysis, and nitrate assimilation differentially nitrate in $A$. thaliana plants challenged with avirulent $P$. syringae pv. tomato during HR (Cecconi et al. 2009). Lozano-Juste and associates (2011) discuss particular aspects and challenges in proteomic approaches related to the identification of tyrosine residues undergoing nitration in their most complete study on the nitroproteome, identifying 127 proteins putatively nitrated in plants. However, only a few reports are available on the identification of in vivo nitration sites in plants, probably because of the low abundance and limited stability of this modification (Galetskiy et al. 2011). The functional consequences of nitration have been studied for only two proteins in plants but, unfortunately, neither protein seems to participate in resistance (Alvarez et al. 2011; Melo et al. 2011). Both studies report inhibition of activity: inhibition by nitration of a specific tyrosine residue of $A$. thaliana $\mathrm{O}$-acetylserine(thiol)lyase A1 (OASA1), which catalyzes the last step of sulfur assimilation (Álvarez et al. 2011), and the nodule glutamine synthase isoenzyme is inhibited by nitration of a specific tyrosine in relation to reduced nitrogen fixation rates (Melo et al. 2011).

\section{Conclusions.}

We described here the pivotal role of $\mathrm{NO}$ as a mediator for plant immunity and current knowledge about its production and signaling. Our understanding of the NO role in defense signaling is still fragmentary, and a strong limitation especially arises because the enzymatic sources of NO in plant-pathogen interactions still remain elusive. Therefore, identification and characterization of NO-producing enzymes in plants during microbe interaction, as well as of genes participating in NO signaling during HR, remain mandatory tasks. To this aim, genetic screenings are still attractive approaches. Screening Arabidopsis mutant collections to identify genes participating in NO signaling during cell death, as well as proteomic approaches or gene hunting by using VIGS to find novel NO-producing enzymes in plants, are promising approaches that should be considered to further improve our knowledge about NO synthesis and signaling during plant defense.

\section{ACKNOWLEDGMENTS}

This work was supported by the Program for Promotion of Basic Research Activities for Innovative Biosciences (PROBRAIN); by a Grant-inAid for Scientific Research on Innovative Areas (23117707) from the Ministry of Education, Culture, Sports, Science and Technology of Japan; and by a grant from the Italian "Ministero dell'Università e della Ricerca" in the framework of the program "Mechanism of plant resistance and response to peroxynitrite".

\section{LITERATURE CITED}

Ali, R., Ma, W., Lemtiri-Chlieh, F., Tsaltas, D., Leng, Q., von Bodman, S., and Berkowitz, G.A. 2007. Death don't have no mercy and neither does calcium: Arabidopsis CYCLIC NUCLEOTIDE GATED CHANNEL2 and innate immunity. Plant Cell 19:1081-1095.

Álvarez, C., Lozano-Juste, J., Romero, L. C., García, I., Gotor, C., and León, J. 2011. Inhibition of Arabidopsis O-Acetylserine(thiol)lyase A1 by tyrosine nitration. J. Biol. Chem. 286:578-586.

Arasimowicz-Jelonek, M., and Floryszak-Wieczorek, J. 2011. Understanding the fate of peroxynitrite in plant cells: From physiology to pathophysiology. Phytochemistry 72:681-688.

Asai, S., and Yoshioka, H. 2009. Nitric oxide as a partner of reactive oxygen species participates in disease resistance to necrotrophic pathogen Botrytis cinerea in Nicotiana benthamiana. Mol. Plant-Microbe Interact. 22:619-629.

Asai, S., Ohta, K., and Yoshioka, H. 2008. MAPK Signaling regulates nitric oxide and NADPH oxidase-dependent oxidative bursts in Nicotiana benthamiana. Plant Cell 20:1390-1406.

Asai, S., Mase, K., and Yoshioka, H. 2010a. A key enzyme for flavin synthesis is required for nitric oxide and reactive oxygen species production in disease resistance. Plant J. 62:911-924.

Asai, S., Mase, K., and Yoshioka, H. 2010b. Role of nitric oxide and reactive oxygen species in disease resistance to necrotrophic pathogens. Plant Signal. Behav. 5:872-874.

Astier, J., Rasul, S., Koen, E., Manzoor, H., Besson-Bard, A., Lamotte, O., Jeandroz, S., Durner, J., Lindermayr, C., and Wendehenne, D. 2011. Snitrosylation: An emerging post-translational protein modification in plants. Plant Sci. 181:527-533.

Astier, J., Besson-Bard, A., Lamotte, O., Bertoldo, J., Bourque, S., Terenzi, H., and Wendehenne, D. 2012. Nitric oxide inhibits the ATPase activity of the chaperone-like AAA+ATPase CDC48, a target for $S$-nitrosylation in cryptogein signaling in tobacco cells. Biochem. J. 447:249-260.

Bayden, A. S., Yakovlev, V. A., Graves, P. R., Mikkelsen, R. B., and Kellogg, G. E. 2011. Factors influencing protein tyrosine nitrationstructure-based predictive models. Free Radic. Biol. Med. 50:749-762.

Belenghi, B., Romero-Puertas, M. C., Vercammen, D., Brackenier, A., Inze, D., Delledonne, M., and Van Breusegem, F. 2007. Metacaspase activity of Arabidopsis thaliana is regulated by $S$-nitrosylation of a critical cysteine residue. J. Biol. Chem. 282:1352-1358.

Benhar, M., Forrester, M. T., Hess, D. T., and Stamler, J. S. 2008. Regulated protein denitrosylation by cytosolic and mitochondrial thioredoxins. Science 320:1050-1054.

Besson-Bard, A., Pugin, A., and Wendehenne, D. 2008. New insights into nitric oxide signaling in plants. Annu. Rev. Plant Biol. 59:21-39.

Bright, J., Desikan, R., Hancock, J. T., Weir, I. S., and Neill, S. J. 2006. ABA-induced NO generation and stomatal closure in Arabidopsis are dependent on $\mathrm{H}_{2} \mathrm{O}_{2}$ synthesis. Plant J. 45:113-122.

Cantrel, C., Vazquez, T., Puyaubert, J., Reze, N., Lesch, M., Kaiser, W. M., Dutilleul, C., Guillas, I., Zachowski, A., and Baudouin, E. 2011. Nitric oxide participates in cold-responsive phosphosphingolipid formation and gene expression in Arabidopsis thaliana. New Phytol. 189:415-427.

Cecconi, D., Orzetti, S., Vandelle, E., Rinalducci, S., Zolla, L., and Delledonne, M. 2009. Protein nitration during defense response in 
Arabidopsis thaliana. Electrophoresis 30:2460-2468

Chaki, M., Fernandez-Ocana, A. M., Valderrama, R., Carreras, A. Esteban, F. J., Luque, F., Gomez-Rodriguez, M. V., Begara-Morales, J. C., Corpas, F. J., and Barroso, J. B. 2009a. Involvement of reactive nitrogen and oxygen species (RNS and ROS) in sunflower-mildew interaction. Plant Cell Physiol. 50:265-279.

Chaki, M., Valderrama, R., Fernandez-Ocana, A. M., Carreras, A., LopezJaramillo, J., Luque, F., Palma, J. M., Pedrajas, J. R., Begara-Morales, J. C., Sanchez-Calvo, B., Gomez-Rodriguez, M. V., Corpas, F. J., and Barroso, J. B. 2009b. Protein targets of tyrosine nitration in sunflower (Helianthus annuus L.) hypocotyls. J. Exp. Bot. 60:4221-4234.

Chen, R., Sun, S., Wang, C., Li, Y., Liang, Y., An, F., Li, C., Dong, H., Yang, X., Zhang, J., and Zuo, J. 2009. The Arabidopsis PARAQUAT RESISTANT2 gene encodes an S-nitrosoglutathione reductase that is a key regulator of cell death. Cell Res. 19:1377-1387.

Clarke, A., Desikan, R., Hurst, R. D., Hancock, J. T., and Neill S. J. 2000. NO way back: Nitric oxide and programmed cell death in Arabidopsis thaliana suspension cultures. Plant J. 24:667-677.

Corpas, F. J., Barroso, J. B., Carreras, A., Valderrama, R., Palma, J. M., Leon, A. M., Sandalio, L. M., and Del Rio, L. A. 2006. Constitutive arginine-dependent nitric oxide synthase activity in different organs of pea seedlings during plant development. Planta 224:246-254.

Corpas, F. J., Palma, J. M., del Rio, L. A., and Barroso, J. B. 2009. Evidence supporting the existence of L-arginine-dependent nitric oxide synthase activity in plants. New Phytol. 184:9-14.

Delledonne, M., Xia, Y., Dixon, R. A., and Lamb, C. 1998. Nitric oxide functions as a signal in plant disease resistance. Nature 394:585-588.

Delledonne, M., Zeier, J., Marocco, A., and Lamb, C. 2001. Signal interactions between nitric oxide and reactive oxygen intermediates in the plant hypersensitive disease resistance response. Proc. Natl. Acad. Sci. U.S.A. 98:13454-13459.

Desikan, R., Griffiths, R., Hancock, J., and Neill, S. 2002. A new role for an old enzyme: Nitrate reductase-mediated nitric oxide generation is required for abscisic acid-induced stomatal closure in Arabidopsis thaliana. Proc. Natl. Acad. Sci. U.S.A. 99:16314-16318.

Dordas, C., Hasinoff, B. B., Rivoal, J., and Hill, R. D. 2004. Class-1 hemoglobins, nitrate and NO levels in anoxic maize cell-suspension cultures. Planta 219:66-72.

Durner, J., Wendehenne, D., and Klessig, D. F. 1998. Defense gene induction in tobacco by nitric oxide, cyclic GMP, and cyclic ADP-ribose. Proc. Natl. Acad. Sci. U.S.A. 95:10328-10333.

Espunya, M. C., De Michele, R., Gomez-Cadenas, A., and Martinez, M. C. 2012. $S$-nitrosoglutathione is a component of wound- and salicylic acidinduced systemic responses in Arabidopsis thaliana. J. Exp. Bot. 63:3219-3227.

Fares, A., Rossignol, M., and Peltier, J.-B. 2011. Proteomics investigation of endogenous $S$-nitrosylation in Arabidopsis. Biochem. Biophys. Res. Commun. 416:331-336.

Feechan, A., Kwon, E., Yun, B. W., Wang, Y., Pallas, J. A., and Loake, G. J. 2005. A central role for $S$-nitrosothiols in plant disease resistance. Proc. Natl. Acad. Sci. U.S.A. 102:8054-8059.

Foresi, N., Correa-Aragunde, N., Parisi, G., Calo, G., Salerno, G., and Lamattina, L. 2010. Characterization of a nitric oxide synthase from the plant kingdom: NO generation from the green alga Ostreococcus tauri is light irradiance and growth phase dependent. Plant Cell 22:3816-3830.

Galetskiy, D., Lohscheider, J. N., Kononikhin, A. S., Popov, I. A., Nikolaev, E. N., and Adamska, I. 2011. Phosphorylation and nitration levels of photosynthetic proteins are conversely regulated by light stress. Plant Mol. Biol. 77:461-473.

Gaupels, F., Kuruthukulangarakoola, G. T., and Durner, J. 2011a. Upstream and downstream signals of nitric oxide in pathogen defence. Curr. Opin. Plant Biol. 14:707-714.

Gaupels, F., Spiazzi-Vandelle, E., Yang, D., and Delledonne, M. 2011b. Detection of peroxynitrite accumulation in Arabidopsis thaliana during the hypersensitive defense response. Nitric Oxide 25:222-228.

Godber, B. L., Doel, J. J., Sapkota, G. P., Blake, D. R., Stevens, C. R., Eisenthal, R., and Harrison, R. 2000. Reduction of nitrite to nitric oxide catalyzed by xanthine oxidoreductase. J. Biol. Chem. 275:7757-7763.

Gorg, B., Qvartskhava, N., Voss, P., Grune, T., Haussinger, D., and Schliess, F. 2007. Reversible inhibition of mammalian glutamine synthetase by tyrosine nitration. FEBS (Fed. Eur. Biochem. Soc.) Lett. 581:84-90.

Gupta, K .J., and Igamberdiev, A. U. 2011. The anoxic plant mitochondrion as a nitrite: NO reductase. Mitochondrion 11:537-543.

Gupta, K. J., Fernie, A. R., Kaiser, W. M., and van Dongen, J. T. 2011. On the origins of nitric oxide. Trends Plant Sci. 16:160-168.

Hardin, S. C., Larue, C. T., Oh, M. H., Jain, V., and Huber, S. C. 2009 Coupling oxidative signals to protein phosphorylation via methionine oxidation in Arabidopsis. Biochem. J. 422:305-312.

Hess, D. T., and Stamler, J. S. 2012. Regulation by $S$-nitrosylation of pro- tein post-translational modification. J. Biol. Chem. 287:4411-4418.

Holtgrefe, S., Gohlke, J., Starmann, J., Druce, S., Klocke, S., Altmann, B., Wojtera, J., Lindermayr, C., and Scheibe, R. 2008. Regulation of plant cytosolic glyceraldehyde 3-phosphate dehydrogenase isoforms by thiol modifications. Physiol. Plant. 133:211-228.

Holzmeister, C., Frohlich, A., Sarioglu, H., Bauer, N., Durner, J., and Lindermayr, C. 2011. Proteomic analysis of defense response of wildtype Arabidopsis thaliana and plants with impaired NO-homeostasis. Proteomics 11:1664-1683.

Horchani, F., Prevot, M., Boscari, A., Evangelisti, E., Meilhoc, E., Bruand, C., Raymond, P., Boncompagni, E., Aschi-Smiti, S., Puppo, A., and Brouquisse, R. 2011. Both plant and bacterial nitrate reductases contribute to nitric oxide production in Medicago truncatula nitrogen-fixing nodules. Plant Physiol. 155:1023-1036.

Isner, J. C., and Maathuis, F. J. 2011. Measurement of cellular cGMP in plant cells and tissues using the endogenous fluorescent reporter FlincG. Plant J. 65:329-34

Kornberg, M. D., Sen, N., Hara, M. R., Juluri, K. R., Nguyen, J. V., Snowman, A. M., Law, L., Hester, L. D., and Snyder, S. H. 2010. GAPDH mediates nitrosylation of nuclear proteins. Nat. Cell Biol. 12:1094-1100.

Kwon, E., Feechan, A., Yun, B. W., Hwang, B. H., Pallas, J. A., Kang, J. G., and Loake, G. J. 2012. AtGSNOR1 function is required for multiple developmental programs in Arabidopsis. Planta 236:887-900.

Laxalt, A. M., Raho, N., Have, A. T., and Lamattina, L. 2007. Nitric oxide is critical for inducing phosphatidic acid accumulation in xylanase-elicited tomato cells. J. Biol. Chem. 282:21160-21168.

Lee, U., Wie, C., Fernandez, B. O., Feelisch, M., and Vierling, E. 2008. Modulation of nitrosative stress by $S$-nitrosoglutathione reductase is critical for thermotolerance and plant growth in Arabidopsis. Plant Cell 20:786-802.

Leitner, M., Vandelle, E., Gaupels, F., Bellin, D., and Delledonne, M. 2009. NO signals in the haze nitric oxide signalling in plant defence. Curr. Opin. Plant Biol. 12:451-458.

Lillo, C., Meyer, C., Lea, U. S., Provan, F., and Oltedal, S. 2004. Mechanism and importance of post-translational regulation of nitrate reductase. J. Exp. Bot. 55:1275-1282.

Lindermayr, C., and Durner, J. 2009. S-nitrosylation in plants: Pattern and function. J. Proteomics 73:1-9.

Lindermayr, C., Sell, S., Muller, B., Leister, D., and Durner, J. 2010. Redox regulation of the NPR1-TGA1 system of Arabidopsis thaliana by nitric oxide. Plant Cell 22:2894-2907.

Liu, L. M., Hausladen, A., Zeng, M., Que, L., Heitman, J., and Stamler, J. S. 2001. A metabolic enzyme for $S$-nitrosothiol conserved from bacteria to humans. Nature 410:490-494.

Lozano-Juste, J., Colom-Moreno, R., and Leon, J. 2011. In vivo protein tyrosine nitration in Arabidopsis thaliana. J. Exp. Bot. 62:3501-3517.

Ma, W., Qi, Z., Smigel, A., Walker, R. K., Verma, R., and Berkowitz, G. A 2009. $\mathrm{Ca}^{2+}$, cAMP, and transduction of non-self perception during plant immune responses. Proc. Natl. Acad. Sci. U.S.A. 106:20995-21000

Meier, S., Madeo, L., Ederli, L., Donaldson, L., Pasqualini, S., and Gehring, C. 2009. Deciphering cGMP signatures and cGMP-dependent pathways in plant defence. Plant Signal Behav. 4:307-309.

Melo, P. M., Silva, L. S., Ribeiro, I., Seabra, A. R., and Carvalho, H. G. 2011. Glutamine synthetase is a molecular target of nitric oxide in root nodules of Medicago truncatula and is regulated by tyrosine nitration. Plant Physiol. 157:1505-1517.

Moche, M., Stremlau, S., Hecht, L., Gobel, C., Feussner, I., and Stohr, C. 2010. Effect of nitrate supply and mycorrhizal inoculation on characteristics of tobacco root plasma membrane vesicles. Planta 231:425-436.

Modolo, L. V., Augusto, O., Almeida, I. M. G., Magalhaes, J. R., and Salgado, I. 2005. Nitrite as the major source of nitric oxide production by Arabidopsis thaliana in response to Pseudomonas syringae. FEBS (Fed. Eur. Biochem. Soc.) Lett. 579:3814-3820.

Modolo, L. V., Augusto, O., Almeida, I. M. G., Pinto-Maglio, C. A. F. Oliveira, H. C., Seligman, K., and Salgado, I. 2006. Decreased arginine and nitrite levels in nitrate reductase-deficient Arabidopsis thaliana plants impair nitric oxide synthesis and the hypersensitive response to Pseudomonas syringae. Plant Sci. 171:34-40.

Mou, Z., Fan, W., and Dong, X. 2003. Inducers of plant systemic acquired resistance regulate NPR1 function through redox changes. Cell 113:935-944.

Mulaudzi, T., Ludidi, N., Ruzvidzo, O., Morse, M., Hendricks, N., Iwuoha, E., and Gehring C. 2011. Identification of a novel Arabidopsis thaliana nitric oxide-binding molecule with guanylate cyclase activity in vitro. FEBS (Fed. Eur. Biochem. Soc.) Lett. 585:2693-2697.

Oliveira, H. C., Justino, G. C., Sodek, L., and Salgado, I. 2009. Amino acid recovery does not prevent susceptibility to Pseudomonas syringae in nitrate reductase double-deficient Arabidopsis thaliana plants. Plant Sci. 176:105-111

Pagnussat, G. C., Lanteri, M. L., Lombardo, M. C., and Lamattina, L. 
2004. Nitric oxide mediates the indole acetic acid induction activation of a mitogen-activated protein kinase cascade involved in adventitious root development. Plant Physiol. 5:279-286.

Park, B. S., Song, J. T., and Seo, H. S. 2011. Arabidopsis nitrate reductase activity is stimulated by the E3 SUMO ligase AtSIZ1. Nat. Commun. 2:400.

Perchepied, L., Balagué, C., Riou, C., Claudel-Renard, C., Rivière, N., Grezes-Besset, B., and Roby, D. 2010. Nitric oxide participates in the complex Interplay of defense-related signaling pathways controlling disease resistance to Sclerotinia sclerotiorum in Arabidopsis thaliana. Mol. Plant-Microbe Interact. 23:846-860.

Planchet, E., Gupta, K. J., Sonoda, M., and Kaiser, W. M. 2005. Nitric oxide (NO) emission from tobacco leaves and cell suspensions: Rate limiting factors and evidence for the involvement of mitochondrial electron transport. Plant J. 41:732-743.

Qi, Z., Verma, R., Gehring, C., Yamaguchi, Y., Zhao, Y., Ryan, C. A., and Berkowitz, G. A. 2010 . $\mathrm{Ca}^{2+}$ signaling by plant Arabidopsis thaliana Pep peptides depends on AtPepR1, a receptor with guanylyl cyclase activity, and cGMP-activated $\mathrm{Ca}^{2+}$ channels. Proc. Natl. Acad. Sci. U.S.A. 7;107:21193-21198.

Radi, R. 2004. Nitric oxide, oxidants, and protein tyrosine nitration. Proc. Natl. Acad. Sci. U.S.A. 101:4003-4008

Raho, N., Ramirez, L., Lanteri, M. L., Gonorazky, G., Lamattina, L., ten Have, A., and Laxalt, A. M. 2011. Phosphatidic acid production in chitosan-elicited tomato cells, via both phospholipase D and phospholipase C/diacylglycerol kinase, requires nitric oxide. J. Plant Physiol. 168:534539.

Rasul, S., Dubreuil-Maurizi, C., Lamotte, O., Koen, E., Poinssot, B., Alcaraz, G., Wendehenne, D., and Jeandroz, S. 2012. Nitric oxide production mediates oligogalacturonides-triggered immunity and resistance to Botrytis cinerea in Arabidopsis thaliana. Plant Cell Environ. 35:1483-1499.

Rockel, P., Strube, F., Rockel, A., Wildt, J., and Kaiser, W. M. 2002. Regulation of nitric oxide (NO) production by plant nitrate reductase in vivo and in vitro. J. Exp. Bot. 53:103-110.

Romero-Puertas, M. C., Laxa, M., Matte, A., Zaninotto, F., Finkemeier, I., Jones, A. M., Perazzolli, M., Vandelle, E., Dietz, K. J., and Delledonne, M. 2007. S-nitrosylation of peroxiredoxin II E promotes peroxynitritemediated tyrosine nitration. Plant Cell 19:4120-4130.

Romero-Puertas, M. C., Campostrini, N., Mattè, A., Righetti, P. G., Perazzolli, M., Zolla, L., Roepstorff, P., and Delledonne, M. 2008. Proteomic analysis of $S$-nitrosylated proteins in Arabidopsis thaliana undergoing hypersensitive response. Proteomics 8:1459-1469.

Rumer, S., Gupta, K. J., and Kaiser, W. M. 2009. Plant cells oxidize hydroxylamines to NO. J. Exp. Bot. 60:2065-2072.

Rusterucci, C., Espunya, M. C., Diaz, M., Chabannes, M., and Martinez, M. C. 2007. $S$-nitrosoglutathione reductase affords protection against pathogens in Arabidopsis, both locally and systemically. Plant Physiol. 143:1282-1292.

Saito, S., Yamamoto-Katou, A., Yoshioka, H., Doke, N., and Kawakita, K. 2006. Peroxynitrite generation and tyrosine nitration in defense responses in tobacco BY-2 cells. Plant Cell Physiol. 47:689-697.

Sen, N., and Snyder, S. H. 2011. Neurotrophin-mediated degradation of histone methyltransferase by $S$-nitrosylation cascade regulates neuronal differentiation. Proc. Natl. Acad. Sci. U.S.A. 108:20178-20183.

Shi, F. M., and Li, Y. Z. 2008. Verticillium dahliae toxins-induced nitric oxide production in Arabidopsis is major dependent on nitrate reductase. BMB Rep. 41:79-85.

Spoel, S. H., and Loake, G. J. 2011. Redox-based protein modifications: The missing link in plant immune signalling. Curr. Opin. Plant Biol. $14: 358-364$

Stamler, J. S., and Hess, D. T. 2010. Nascent nitrosylases. Nat. Cell Biol. 12:1024-1026.

Stohr, C., Strube, F., Marx, G., Ullrich, W. R., and Rockel, P. 2001. A plasma membrane-bound enzyme of tobacco roots catalyses the formation of nitric oxide from nitrite. Planta 212:835-841.

Sueldo, D. J., Foresi, N. P., Casalongué, C. A., Lamattina, L., and Laxalt, A. M. 2010. Phosphatidic acid formation is required for extracellular ATP-mediated nitric oxide production in suspension-cultured tomato cells. New Phytol. 185:909-916.

Sun, J., Zhang, C. L., Deng, S. R., Lu, C. F., Shen, X., Zhou, X. Y., Zheng, X. J., Hu, Z. M., and Chen, S. L. 2012. An ATP signalling pathway in plant cells: Extracellular ATP triggers programmed cell death in Populus euphratica. Plant Cell Environ. 35:893-916.

Tada, Y., Spoel, S. H., Pajerowska-Mukhtar, K., Mou, Z., Song, J., Wang, C., Zuo, J., and Dong, X. 2008. Plant immunity requires conformational charges of NPR1 via $S$-nitrosylation and thioredoxins. Science 321:952956.
Terrile, M. C., Paris, R., Calderon-Villalobos, L. I., Iglesias, M. J. Lamattina, L., Estelle, M., and Casalongue, C. A. 2012. Nitric oxide influences auxin signaling through $S$-nitrosylation of the Arabidopsis TRANSPORT INHIBITOR RESPONSE 1 auxin receptor. Plant J. 70:492-500.

Torres, M. A., Jones, J. D., and Dangl, J. L. 2005. Pathogen-induced, NADPH oxidase-derived reactive oxygen intermediates suppress spread of cell death in Arabidopsis thaliana. Nat. Genet. 37:1130-1134.

Tun, N. N., Santa-Catarina, C., Begum, T., Silveira, V., Handro, W., Floh, E. I. S., and Scherer, G. F. E. 2006. Polyamines induce rapid biosynthesis of nitric oxide (NO) in Arabidopsis thaliana seedlings. Plant Cell Physiol. 47:346-354.

Vandelle, E., and Delledonne, M. 2011. Peroxynitrite formation and function in plants. Plant Sci. 181:534-539.

van der Linde, K., Gutsche, N., Leffers, H. M., Lindermayr, C., Muller, B. Holtgrefe, S., and Scheibe, R. 2011. Regulation of plant cytosolic aldolase functions by redox-modifications. Plant Physiol. Biochem. 49:946-957.

Wang, P., Du, Y., Li, Y., Ren, D., and Song, C.-P. 2010. Hydrogen peroxide-mediated activation of MAP kinase 6 modulates nitric oxide biosynthesis and signal transduction in Arabidopsis. Plant Cell 22:2981-2998.

Wang, P., Du, Y., and Song, C. P. 2011. Phosphorylation by MPK6: A conserved transcriptional modification mediates nitrate reductase activation and NO production? Plant Signal. Behav. 6:889-891.

Wang, Y. Q., Feechan, A., Yun, B. W., Shafiei, R., Hofmann, A., Taylor, P., Xue, P., Yang, F. Q., Xie, Z. S., Pallas, J. A., Chu, C. C., and Loake, G. J. 2009. S-nitrosylation of AtSABP3 antagonizes the expression of plant immunity. J. Biol. Chem. 284:2131-2137.

Wawer, I., Bucholc, M., Astier, J., Anielska-Mazur A., Dahan, J., Kulik, A., Wysłouch-Cieszynska, A., Zareba-Kozioł, M., Krzywinska, E., Dadlez, M., Dobrowolska, G., and Wendehenne, D. 2010. Regulation of Nicotiana tabacum osmotic stress-activated protein kinase and its cellular partner GAPDH by nitric oxide in response to salinity. Biochem. J. 429:73-83.

Weerapana, E., Wang, C., Simon, G. M., Richter, F., Khare, S., Dillon, M. B., Bachovchin, D. A., Mowen, K., Baker, D., and Cravatt, B. F. 2010 Quantitative reactivity profiling predicts functional cysteines in proteomes. Nature 468:790-795.

Wendehenne, D., Pugin, A., Klessig, D. F., and Durner, J. 2001. Nitric oxide: Comparative synthesis and signaling in animal and plant cells. Trends Plant Sci. 6:177-183.

Wilkinson, J. Q., and Crawford, N. M. 1993. Identification and characterization of a chlorate-resistant mutant of Arabidopsis thaliana with mutations in both nitrate reductase structural genes NIA1 and NIA2. Mol. Gen. Genet. 239:289-297.

Wimalasekera, R., Villar, C., Begum, T., and Scherer, G. F. 2011. COPPER AMINE OXIDASE1 (CuAO1) of Arabidopsis thaliana contributes to abscisic acid- and polyamine-induced nitric oxide biosynthesis and abscisic acid signal transduction. Mol. Plant 4:663-678.

Wu, Y., Zhang D., Chu, J. Y., Boyle, P., Wang, Y., Brindle, I. D., De, Luca V., and Després, C. 2012. The Arabidopsis NPR1 protein is a receptor for the plant defense hormone salicylic acid. Cell Rep. 8:639-647.

Yamamoto-Katou, A., Katou, S., Yoshioka, H., Doke, N., and Kawakita, K. 2006. Nitrate reductase is responsible for elicitin-induced nitric oxide production in Nicotiana benthamiana. Plant Cell Physiol. 47:726735.

Yamasaki, H., Sakihama, Y., and Takahashi, S. 1999. An alternative pathway for nitric oxide production in plants: New features of an old enzyme. Trends Plant Sci. 4:128-129.

Yoshioka, H., Mase, K., Yoshioka, M., Kobayashi, M., and Asai, S. 2011. Regulatory mechanisms of nitric oxide and reactive oxygen species generation and their role in plant immunity. Nitric Oxide 25:216-221.

Yun, B. W., Spoel, S. H., and Loake, G. J. 2011. Synthesis of and signalling by small, redox active molecules in the plant immune response. Biochim. Biophys. Acta 1820:770-776.

Yun, B. W., Feechan, A., Yin, M., Saidi, N. B., Le Bihan, T., Yu, M., Moore, J. W., Kang, J. G., Kwon, E., Spoel, S. H., Pallas, J. A., and Loake, G. J. 2012. $S$-nitrosylation of NADPH oxidase regulates cell death in plant immunity. Nature 478:264-268.

Zhang, H., Li, D., Wang, M., Liu, J., Teng, W., Cheng, B., Dong, S., Zheng, X., and Zhang Z. 2012. Nicotiana benthamiana MAPK cascade and WRKY transcription factor participate in Nep1 $1_{\mathrm{Mo}}$-triggered plant responses. Mol. Plant-Microbe Interact. 25:1639-1653.

Zhang, Y. H., and Hogg, N. 2004. The mechanism of transmembrane $S$-nitrosothiol transport. Proc. Natl. Acad. Sci. U.S.A. 101:7891-7896.

Zhao, M. G., Chen, L., Zhang, L. L., and Zhang, W. H. 2009. Nitric reductase-dependent nitric oxide production is involved in cold acclimation and freezing tolerance in Arabidopsis. Plant Physiol. 151:755-767. 